\title{
GW23-e0853 PROGNOSTIC VALUE OF CIRCULATING CATESTATIN LEVELS ON HOSPITALISED PATIENTS WITH ACUTE MYOCARDIAL INFARCTION
}

doi:10.1136/heartjnl-2012-302920j.34

Pei Zhi-qiang, Pei Zhi-qiang. Taiyuan Central Hospital

Objectives To evaluate prognostic value of circulating catestatin (CST) on hospitalised patients with acute myocardial infarction.

Methods The data of 125 patients with AMI were collected from the Second Hospital of Shanxi Medical University and Taiyuan central hospital during the period November 2010 to July 2011. Recording age, gender, body mass index, smoking history, past medical history, blood pressure, heart rate, Killip class at the time of admission, various biochemical index, cardiac ultrasonography the incidence of malignant events etc. The malignant events of this research included malignant arrhythmia, heart failure, angina or reinfarction, death. The patients were categorised into 4 groups according to CST $(\mathrm{pg} / \mathrm{ml})$ quartile. Clinical features, therapeutic approaches and the incidence of heart failure, malignant arrhythmias and death during hospitalisation were compared among groups. At the same time the patients were grouped into whether AMI with heart failure, malignant arrhythmias, if AMI with heart failure, were divided into three subunits according to Killip class. CST, NE, NT-proBNP were compared among groups. Multivariate logistic regression analysis was applied to determine the association between risk factors and in-hospital malignant events occurred. Receiver-operator characteristic (ROC) curve was performed to evaluate the power of CST on predicting in-hospital malignant events occurred.

\section{Results}

1. Clinical features: Patients with higher CST values were more likely to be older, to have lower body mass index and ejection fraction; to have higher white blood cell count, CysC, hs-CRP, NE, NT-proBNP value;to more likely have past history of angina, past history of diabetes mellitus; to more diuretic users $(p<0.05)$.

2. In-hospital malignant events: Higher CST levels were associated with increased risk of heart failure, malignant arrhythmias and death $(p<0.05)$. The area under the ROC curve of CST was $0.729,95 \%$ CI 0.640 to 0.817 , when CST $=72.65$ (pg/ml), had the best sensitivity $(92.7 \%)$ and specificity $(72.9 \%)$. After multivariate adjustment age, CST, NT-proBNP remained to be independent risk factors for increased in-hospital malignant events.

3. AMI with heart failure: CST and NT-proBNP levels were higher in AMI with heart failure $(p<0.01)$.CST, NE, NT-proBNP levels were increased in proportion to increasing Killip grades (II-IV). The area under the ROC curve of CST was $0.714,95 \%$ CI 0.638 to 0.791 , when $\mathrm{CST}=70.08(\mathrm{pg} / \mathrm{ml})$, had the best sensitivity $(95.6 \%)$ and specificity (70.6\%).After multivariate adjustment age, history of heart failure, hs-CRP, CST, NT-proBNP remained to be independent risk factors for increased in-hospital heart failure occurred.

4. AMI with malignant arrhythmias: CST, NE, NT-proBNP levels were higher in AMI with malignant arrhythmias $(p<0.05)$. The 


\section{ABSTRACTS}

area under the ROC curve of CST was 0.725 , 95\% CI 0.629 to 0.820 , when CST $=70.35(\mathrm{pg} / \mathrm{ml})$, had the best sensitivity (96.9\%) and specificity (78.7\%). After multivariate adjustment pathoglycemia, CST remained to be independent risk factors for increased in-hospital malignant arrhythmias occurred.

\section{Conclusions}

1. The plasma CST level is an independent predictor to in-hospital malignant events and to some extent CST provides incremental prognostic information to conventional cardiovascular risk marker for patients with AMI.

2. The plasma CST level is an independent risk factor for heart failure occurred during hospitalisation in patients with AMI.

3. The plasma CST level is significant associated with in-hospital malignant arrhythmias occurred. 